\title{
CONICAL STRUCTURE FOR SHRINKING RICCI SOLITONS
}

\author{
OVIDIU MUNTEANU AND JIAPING WANG
}

\begin{abstract}
For a shrinking Ricci soliton with Ricci curvature convergent to zero at infinity, it is shown that it must be asymptotically conical.
\end{abstract}

\section{INTRODUCTION}

The purpose of this paper is to show that a shrinking gradient Ricci soliton must be smoothly asymptotic to a cone if its Ricci curvature goes to zero at infinity. Recall that a gradient shrinking Ricci soliton is a Riemannian manifold $\left(M^{n}, g\right)$ for which there exists a potential function $f$ such that

$$
\operatorname{Ric}+\operatorname{Hess}(f)=\frac{1}{2} g,
$$

where Ric is the Ricci curvature of $M$ and Hess $(f)$ the Hessian of $f$. Aside from its own interest as generalization of Einstein manifolds, Ricci solitons are important in the study of the Ricci flows. Indeed, one easily verifies (see [10]) that $g(t)=$ $(1-t) \phi_{t}^{*} g$, for $-\infty<t<1$, is a solution to the Ricci flow

$$
\begin{aligned}
\frac{\partial}{\partial t} g(t) & =-2 \operatorname{Ric}(g(t)) \\
g(0) & =g
\end{aligned}
$$

for a suitably chosen family of diffeomorphisms $\phi_{t}$ on $M$ with $\phi_{0}=i d$. So shrinking Ricci solitons may be regarded as self-similar solutions to the Ricci flows. It has been shown in 13 , that the blow-ups around a type-I singularity point always converge to nontrivial gradient shrinking Ricci solitons. Therefore, it would be very desirable to understand and even classify shrinking Ricci solitons.

In the case dimension $n=2$, according to [15, the only examples are either the sphere $\mathbb{S}^{2}$ or the Gaussian soliton, Euclidean space $\mathbb{R}^{2}$ together with potential function $f(x)=\frac{1}{4}|x|^{2}$. For dimension $n=3$, improving upon the breakthrough of Perelman 24, Naber 22, Ni and Wallach 23, and Cao, Chen and Zhu [5] have concluded that a three dimensional shrinking gradient Ricci soliton must be a quotient of the sphere $\mathbb{S}^{3}$, or $\mathbb{R}^{3}$, or $\mathbb{S}^{2} \times \mathbb{R}$.

For high dimensional shrinking Ricci solitons, examples other than the sphere and Gaussian soliton (and their products) have been constructed by 2, 16, 27, 14, 11. This certainly indicates that it would be more complicated, if at all possible, to obtain a complete classification. Under some auxiliary conditions on the full curvature tensor, partial classification results have been established. In 22, Naber has shown that a four dimensional complete shrinking Ricci soliton of bounded nonnegative curvature operator must be a quotient of $\mathbb{R}^{k} \times \mathbb{S}^{4-k}$ with $k=0,1,2$. A theorem of Böhm and Wilking [1] implies that a compact shrinking Ricci soliton of any dimension with positive curvature operator must be a spherical space form. 
Also, shrinking gradient Ricci solitons of vanishing Weyl tensor have been classified and must be finite quotients of sphere $\mathbb{S}^{n}$, or $\mathbb{R}^{n}$, or $\mathbb{S}^{n-1} \times \mathbb{R}$ (see [28, 12, 25, 8, 19]). More generally, in a recent work [6], Cao and Chen have shown that a Bachflat gradient shrinking Ricci soliton is either Einstein, or a finite quotient of the Gaussian shrinking soliton $\mathbb{R}^{n}$, or a finite quotient of $N^{n-1} \times \mathbb{R}$, where $N^{n-1}$ is an Einstein manifold of positive scalar curvature. We refer the reader to the two surveys 3 , 4, for more results and details.

In another direction, Kotschwar and Wang [17] have recently shown that two shrinking Ricci solitons $C^{2}$ asymptotic to the same cone must be isometric. Here, by a cone, we mean a manifold $[0, \infty) \times \Sigma$ endowed with Riemannian metric $g_{c}=$ $d r^{2}+r^{2} g_{\Sigma}$, where $\left(\Sigma, g_{\Sigma}\right)$ is a closed $(n-1)$-dimensional Riemannian manifold. Denote $E_{R}=(R, \infty) \times \Sigma$ for $R \geq 0$ and define the dilation by $\lambda$ to be the map $\rho_{\lambda}: E_{0} \rightarrow E_{0}$ given by $\rho_{\lambda}(r, \sigma)=(\lambda r, \sigma)$. A Riemannian manifold $(M, g)$ is said to be $C^{k}$ asymptotic to the cone $\left(E_{0}, g_{c}\right)$ if, for some $R>0$, there is a diffeomorphism $\Phi: E_{R} \rightarrow M \backslash \Omega$ such that $\lambda^{-2} \rho_{\lambda}^{*} \Phi^{*} g \rightarrow g_{c}$ as $\lambda \rightarrow \infty$ in $C_{l o c}^{k}\left(E_{0}, g_{c}\right)$, where $\Omega$ is a compact subset of $M$.

In view of their result, it becomes an interesting question to determine when a shrinking Ricci soliton is asymptotically conical. In our recent work 20, we have shown that this is the case for four dimensional shrinking gradient Ricci solitons with scalar curvature converging to 0 at infinity. This result depends on the fact that the full curvature tensor $\mathrm{Rm}$ of a four dimensional soliton is controlled by its scalar curvature $S$ alone, that is, $|\mathrm{Rm}| \leq c|\mathrm{Ric}| \leq c S$. While it remains to be seen whether such an estimate is true for high dimensional shrinking Ricci solitons, by imposing assumption on the Ricci curvature instead, we manage to obtain a parallel result as well.

Theorem 1.1. Let $(M, g, f)$ be a gradient shrinking Ricci soliton of dimension $n$ with Ricci curvature convergent to zero at infinity. Then $(M, g, f)$ is $C^{k}$ asymptotic to a cone for all $k$.

Consequently, the classification problem for such solitons is reduced to the one for cones.

Essential to the proof of Theorem 1.1 is a quadratic decay estimate for the Riemann curvature $|\mathrm{Rm}|$. Once this is available, together with Shi's 26] derivative estimates of $\mathrm{Rm}$, it is then straightforward to conclude that $(M, g)$ is asymptotically conical [17. As demonstrated in 20, such a decay estimate follows from a maximum principle argument provided that the Riemann curvature tensor $\mathrm{Rm}$ converges to zero at infinity. So the heart of the proof is to conclude from Ric converging to 0 that $\mathrm{Rm}$ goes to 0 as well. Here, we are very much inspired by the work of 21, where it says that for a shrinking Ricci soliton, its Riemann curvature is at most of polynomial growth if its Ricci curvature is bounded. However, we would like to point out that our argument differs significantly from [21] in terms of technical details.

We remark that our argument only requires the Ricci curvature being sufficiently small outside a compact set. More precisely, Theorem 1.1 continues to hold if one assumes instead that $|\mathrm{Ric}| \leq \delta$ near the infinity of $M$ for some positive constant $\delta$ depending only on the dimension $n$. 


\section{Curvature estimates}

In this section, we prove Theorem 1.1. We continue to denote by $(M, g, f)$ an $n$-dimensional shrinking Ricci soliton with potential function $f$.

Let us recall the following important identities

$$
\begin{aligned}
\nabla_{k} R_{j k} & =R_{j k} f_{k}=\frac{1}{2} \nabla_{j} S \\
\nabla_{l} R_{i j k l} & =R_{i j k l} f_{l}=\nabla_{j} R_{k i}-\nabla_{i} R_{k j} .
\end{aligned}
$$

As observed in [15, this implies $S+|\nabla f|^{2}=f$ by adding a suitable constant to $f$. Since $S \geq 0$ by [9], we have $|\nabla f|^{2} \leq f$.

Also, denoting $\Delta_{f}=\Delta-\langle\nabla f, \nabla\rangle$, we have

$$
\begin{aligned}
\Delta_{f} R_{i j} & =R_{i j}-2 R_{i k j l} R_{k l} \\
\Delta_{f} \mathrm{Rm} & =\mathrm{Rm}+\mathrm{Rm} * \mathrm{Rm}
\end{aligned}
$$

Let us denote

$$
D(r):=\{x \in M: f(x) \leq r\} .
$$

Notice that $D(r)$ is always compact as by [7] there exists constant $c$ such that

$$
\frac{1}{4} r^{2}(x)-c r(x) \leq f(x) \leq \frac{1}{4} r^{2}(x)+c r(x) \text { for } r(x) \geq 1 .
$$

Here $r(x)$ is the distance from $x$ to a fixed point $x_{0} \in M$. Also, recall ( see [7]) that the volume $V(r)$ of $D(r)$ satisfies

$$
V(r) \leq c r^{\frac{n}{2}}
$$

We define the cut-off $\phi$ with support in $D(r)$ by

$$
\phi(x)=\left\{\begin{array}{clc}
\frac{1}{r}(r-f(x)) & \text { if } & x \in D(r) \\
0 & \text { if } & x \in M \backslash D(r)
\end{array}\right.
$$

Let us choose $r_{0}>0$ large enough so that $f \geq 1$ and

$$
|\mathrm{Ric}| \leq \frac{1}{p^{5}} \text { on } M \backslash D\left(r_{0}\right) .
$$

In particular, since

we have

$$
\operatorname{Ric}+\operatorname{Hess}(f)=\frac{1}{2} g
$$

$$
\operatorname{Hess}(f) \geq \frac{1}{3} g \text { on } M \backslash D\left(r_{0}\right) .
$$

We fix $p \geq 8 n$ large enough depending only on dimension $n$ and let $q$ and $a$ be constants satisfying

$$
q \geq 2 p+3 \text { and } a \leq \frac{1}{4} p .
$$

Throughout the paper, unless otherwise indicated, we will use $C$ to denote constants that may depend on the geometry of $D\left(r_{0}\right), c$ constants depending only on dimension $n$ but independent of $p$, and $c(p)$ constants depending on $p$. These constants may change from line to line. We first prove the following lemma. 
Lemma 2.1. Let $(M, g, f)$ be a gradient shrinking Ricci soliton of dimension $n$ with $\lim _{x \rightarrow \infty}|\operatorname{Ric}|(x)=0$. Then there exist positive constants $c, C$ and $c(p)$ such that for $\alpha \in\{0,1\}$,

$$
\begin{aligned}
\int_{M}|\operatorname{Rm}|^{p} f^{a} \phi^{q} \leq & \frac{c}{p} \int_{M}|\operatorname{Rm}|^{p-1+\alpha} f^{a} \phi^{q}+\frac{c}{p} \int_{M}|\operatorname{Rm}|^{p+\alpha} f^{a} \phi^{q} \\
& +c(p) \int_{M}|\operatorname{Ric}|^{p} f^{a} \phi^{q}+C .
\end{aligned}
$$

Proof. Integrating by parts and using that $\Delta f \leq \frac{n}{2}$, we get

$$
\begin{aligned}
-\frac{n}{2} \int_{M}|\mathrm{Rm}|^{p} f^{a} \phi^{q} \leq & -\int_{M}|\mathrm{Rm}|^{p}(\Delta f) f^{a} \phi^{q} \\
= & \int_{M}\left\langle\nabla|\mathrm{Rm}|^{p}, \nabla f\right\rangle f^{a} \phi^{q} \\
& +a \int_{M}|\mathrm{Rm}|^{p}|\nabla f|^{2} f^{a-1} \phi^{q} \\
& +\int_{M}|\mathrm{Rm}|^{p} f^{a}\left\langle\nabla f, \nabla \phi^{q}\right\rangle \\
\leq & \int_{M}\left\langle\nabla|\mathrm{Rm}|^{p}, \nabla f\right\rangle f^{a} \phi^{q}+a \int_{M}|\mathrm{Rm}|^{p} f^{a} \phi^{q},
\end{aligned}
$$

where in the last line we have used that $\left\langle\nabla f, \nabla \phi^{q}\right\rangle \leq 0$. Therefore, by Bianchi identities we obtain that

$$
\begin{aligned}
-\left(a+\frac{n}{2}\right) \int_{M}|\mathrm{Rm}|^{p} f^{a} \phi^{q} & \leq \int_{M}\left\langle\nabla|\mathrm{Rm}|^{p}, \nabla f\right\rangle f^{a} \phi^{q} \\
& =p \int_{M} f_{h}\left(\nabla_{h} R_{i j k l}\right) R_{i j k l}|\mathrm{Rm}|^{p-2} f^{a} \phi^{q} \\
& =2 p \int_{M} f_{h}\left(\nabla_{l} R_{i j k h}\right) R_{i j k l}|\mathrm{Rm}|^{p-2} f^{a} \phi^{q} .
\end{aligned}
$$

It follows through integration by parts that

$$
\begin{aligned}
& -\left(a+\frac{n}{2}\right) \int_{M}|\mathrm{Rm}|^{p} f^{a} \phi^{q} \\
\leq & -2 p \int_{M} R_{i j k h} f_{h l} R_{i j k l}|\mathrm{Rm}|^{p-2} f^{a} \phi^{q} \\
& -2 p \int_{M} R_{i j k h} f_{h}\left(\nabla_{l} R_{i j k l}\right)|\mathrm{Rm}|^{p-2} f^{a} \phi^{q} \\
& -2 p \int_{M} R_{i j k h} f_{h} R_{i j k l}\left(\nabla_{l}|\mathrm{Rm}|^{p-2}\right) f^{a} \phi^{q} \\
& -2 a p \int_{M} R_{i j k h} f_{h} R_{i j k l} f_{l}|\mathrm{Rm}|^{p-2} f^{a-1} \phi^{q} \\
& +\frac{2 p q}{r} \int_{M} R_{i j k h} f_{h} R_{i j k l} f_{l}|\mathrm{Rm}|^{p-2} f^{a} \phi^{q-1} .
\end{aligned}
$$

Note that on $M \backslash D\left(r_{0}\right)$, by (2.6),

$$
-R_{i j k h} f_{h l} R_{i j k l} \leq-\frac{1}{3}|\mathrm{Rm}|^{2} .
$$


Together with (2.1), it results from (2.8) that

$$
\begin{aligned}
\frac{p}{3} \int_{M}|\mathrm{Rm}|^{p} f^{a} \phi^{q} \leq & -2 p \int_{M} R_{i j k h} f_{h} R_{i j k l}\left(\nabla_{l}|\mathrm{Rm}|^{p-2}\right) f^{a} \phi^{q} \\
& +\frac{2 p q}{r} \int_{M}\left|R_{i j k h} f_{h}\right|^{2}|\mathrm{Rm}|^{p-2} f^{a} \phi^{q-1} \\
& +C .
\end{aligned}
$$

By (2.1) again, we have

$$
\begin{aligned}
& -2 p \int_{M} R_{i j k h} f_{h} R_{i j k l}\left(\nabla_{l}|\mathrm{Rm}|^{p-2}\right) f^{a} \phi^{q} \\
\leq & c p^{2} \int_{M}|\nabla \mathrm{Ric}||\nabla \mathrm{Rm}||\mathrm{Rm}|^{p-2} f^{a} \phi^{q} .
\end{aligned}
$$

For $\alpha \in\{0,1\}$, it follows from (2.9) that

$$
\begin{aligned}
\int_{M}|\mathrm{Rm}|^{p} f^{a} \phi^{q} \leq & c p^{3} \int_{M}|\nabla \mathrm{Ric}|^{2}|\mathrm{Rm}|^{p-1-\alpha} f^{a} \phi^{q} \\
& +\frac{c}{p} \int_{M}|\nabla \mathrm{Rm}|^{2}|\mathrm{Rm}|^{p-3+\alpha} f^{a} \phi^{q} . \\
& +\frac{c p}{r} \int_{M}\left|R_{i j k h} f_{h}\right|^{2}|\mathrm{Rm}|^{p-2} f^{a} \phi^{q-1}+C .
\end{aligned}
$$

We now estimate the first term on the right side of (2.10). Note that (2.2) implies

$$
\begin{aligned}
\Delta_{f}\left(|\mathrm{Ric}|^{2}|\mathrm{Rm}|^{p-1-\alpha}\right)= & \left(\Delta_{f}|\mathrm{Ric}|^{2}\right)|\mathrm{Rm}|^{p-1-\alpha}+|\operatorname{Ric}|^{2} \Delta_{f}|\operatorname{Rm}|^{p-1-\alpha} \\
& +2\left\langle\nabla|\operatorname{Ric}|^{2}, \nabla|\operatorname{Rm}|^{p-1-\alpha}\right\rangle \\
\geq & 2|\nabla \operatorname{Ric}|^{2}|\operatorname{Rm}|^{p-1-\alpha}-c p|\operatorname{Ric}|^{2}|\operatorname{Rm}|^{p-\alpha} \\
& -c p|\nabla \operatorname{Ric}||\nabla \operatorname{Rm}||\operatorname{Ric}||\operatorname{Rm}|^{p-2-\alpha} .
\end{aligned}
$$

Consequently, we get

$$
\begin{aligned}
& 2 \int_{M}|\nabla \mathrm{Ric}|^{2}|\mathrm{Rm}|^{p-1-\alpha} f^{a} \phi^{q} \\
\leq & \int_{M} \Delta_{f}\left(|\operatorname{Ric}|^{2}|\mathrm{Rm}|^{p-1-\alpha}\right) f^{a} \phi^{q}+c p \int_{M}|\operatorname{Ric}|^{2}|\operatorname{Rm}|^{p-\alpha} f^{a} \phi^{q} \\
& +c p \int_{M}|\nabla \operatorname{Ric}||\nabla \mathrm{Rm}||\operatorname{Ric}||\operatorname{Rm}|^{p-2-\alpha} f^{a} \phi^{q} .
\end{aligned}
$$

The last term in (2.11) can be estimated by

$$
\begin{aligned}
& 2 \int_{M}|\nabla \mathrm{Ric}||\nabla \mathrm{Rm}||\mathrm{Ric}||\mathrm{Rm}|^{p-2-\alpha} f^{a} \phi^{q} \\
\leq & \frac{1}{p^{5}} \int_{M}|\nabla \mathrm{Rm}|^{2}|\mathrm{Rm}|^{p-3+\alpha} f^{a} \phi^{q} \\
& +p^{5} \int_{M}|\nabla \mathrm{Ric}|^{2}|\mathrm{Ric}|^{2}|\mathrm{Rm}|^{p-1-3 \alpha} f^{a} \phi^{q} .
\end{aligned}
$$


We claim that

$$
\begin{aligned}
& p^{5} \int_{M}|\nabla \mathrm{Ric}|^{2}|\mathrm{Ric}|^{2}|\mathrm{Rm}|^{p-1-3 \alpha} f^{a} \phi^{q} \\
\leq & \frac{1}{p^{2}} \int_{M}|\nabla \mathrm{Ric}|^{2}|\mathrm{Rm}|^{p-1-\alpha} f^{a} \phi^{q} \\
& +c(p) \alpha \int_{M}|\nabla \mathrm{Ric}|^{2}|\mathrm{Ric}|^{p-2} f^{a} \phi^{q}+C .
\end{aligned}
$$

Indeed, for $\alpha=0$ this is obvious by (2.5), whereas for $\alpha=1$, it follows immediately from Young's inequality. Plugging (2.13) into (2.12), we get

$$
\begin{aligned}
& 2 \int_{M}|\nabla \mathrm{Ric}||\nabla \mathrm{Rm}||\mathrm{Ric}||\mathrm{Rm}|^{p-2-\alpha} f^{a} \phi^{q} \\
\leq & \frac{1}{p^{5}} \int_{M}|\nabla \mathrm{Rm}|^{2}|\mathrm{Rm}|^{p-3+\alpha} f^{a} \phi^{q} \\
& +\frac{1}{p^{2}} \int_{M}|\nabla \mathrm{Ric}|^{2}|\mathrm{Rm}|^{p-1-\alpha} f^{a} \phi^{q} \\
& +c(p) \alpha \int_{M}|\nabla \mathrm{Ric}|^{2}|\mathrm{Ric}|^{p-2} f^{a} \phi^{q}+C .
\end{aligned}
$$

We now estimate

$$
\begin{aligned}
\int_{M}|\nabla \mathrm{Ric}|^{2}|\mathrm{Ric}|^{p-2} f^{a} \phi^{q} \leq & \int_{M}\left(\Delta|\mathrm{Ric}|^{2}\right)|\mathrm{Ric}|^{p-2} f^{a} \phi^{q} \\
& -\int_{M}\left\langle\nabla f, \nabla|\mathrm{Ric}|^{2}\right\rangle|\mathrm{Ric}|^{p-2} f^{a} \phi^{q} \\
& +c \int_{M}|\mathrm{Rm}||\mathrm{Ric}|^{p} f^{a} \phi^{q} \\
\leq & -a \int_{M}\left\langle\nabla f, \nabla|\mathrm{Ric}|^{2}\right\rangle|\mathrm{Ric}|^{p-2} f^{a-1} \phi^{q} \\
& +\frac{q}{r} \int_{M}\left\langle\nabla f, \nabla|\mathrm{Ric}|^{2}\right\rangle|\mathrm{Ric}|^{p-2} f^{a} \phi^{q-1} \\
& -\int_{M}\left\langle\nabla f, \nabla|\mathrm{Ric}|^{2}\right\rangle|\mathrm{Ric}|^{p-2} f^{a} \phi^{q} \\
& +c \int_{M}|\mathrm{Rm}||\mathrm{Ric}|^{p} f^{a} \phi^{q} .
\end{aligned}
$$

Note that 


$$
\begin{aligned}
-\int_{M}\left\langle\nabla f, \nabla|\operatorname{Ric}|^{2}\right\rangle|\operatorname{Ric}|^{p-2} f^{a} \phi^{q}= & -\frac{2}{p} \int_{M}\left\langle\nabla f, \nabla|\operatorname{Ric}|^{p}\right\rangle f^{a} \phi^{q} \\
= & \frac{2}{p} \int_{M}|\operatorname{Ric}|^{p}\left(\Delta f+a|\nabla f|^{2} f^{-1}\right) f^{a} \phi^{q} \\
& -\frac{2 q}{p r} \int_{M}|\operatorname{Ric}|^{p} f^{a} \phi^{q-1} \\
\leq & c \int_{M}|\operatorname{Ric}|^{p} f^{a} \phi^{q} .
\end{aligned}
$$

A similar argument also implies

$$
\begin{aligned}
\frac{q}{r} \int_{M}\left\langle\nabla f, \nabla|\operatorname{Ric}|^{2}\right\rangle|\operatorname{Ric}|^{p-2} f^{a} \phi^{q-1} & \leq \frac{c p}{r} \int_{M}|\operatorname{Ric}|^{p} f^{a} \phi^{q-2}+C \\
& \leq c \int_{M}|\operatorname{Ric}|^{p} f^{a} \phi^{q}+\frac{c(p)}{r^{\frac{q}{2}}} \int_{D(r)}|\operatorname{Ric}|^{p} f^{a}+C \\
& \leq c \int_{M}|\operatorname{Ric}|^{p} f^{a} \phi^{q}+C
\end{aligned}
$$

in view of (2.4) and $|\mathrm{Ric}| \leq C$.

It is then easy to see that these estimates and (2.15) imply that

$$
\begin{aligned}
c(p) \int_{M}|\nabla \mathrm{Ric}|^{2}|\operatorname{Ric}|^{p-2} f^{a} \phi^{q} \leq & c(p) \int_{M}|\operatorname{Ric}|^{p} f^{a} \phi^{q} \\
& +c(p) \int_{M}|\operatorname{Rm}||\operatorname{Ric}|^{p} f^{a} \phi^{q}+C \\
\leq & \frac{1}{p^{5}} \int_{M}|\operatorname{Rm}|^{p} f^{a} \phi^{q} \\
& +c(p) \int_{M}|\operatorname{Ric}|^{p} f^{a} \phi^{q}+C .
\end{aligned}
$$

Using (2.16) and (2.14) we get that

$$
\begin{aligned}
& \int_{M}|\nabla \mathrm{Ric}||\nabla \mathrm{Rm}||\mathrm{Ric}||\mathrm{Rm}|^{p-2-\alpha} f^{a} \phi^{q} \\
\leq & \frac{1}{p^{5}} \int_{M}|\nabla \mathrm{Rm}|^{2}|\mathrm{Rm}|^{p-3+\alpha} f^{a} \phi^{q} \\
& +\frac{1}{p^{2}} \int_{M}|\nabla \mathrm{Ric}|^{2}|\mathrm{Rm}|^{p-1-\alpha} f^{a} \phi^{q} \\
& +\frac{1}{p^{5}} \int_{M}|\mathrm{Rm}|^{p} f^{a} \phi^{q}+c(p) \int_{M}|\operatorname{Ric}|^{p} f^{a} \phi^{q}+C .
\end{aligned}
$$

We estimate the first term in (2.11) as follows. First, observe that integration by parts yields 


$$
\begin{aligned}
& \int_{M} \Delta_{f}\left(|\mathrm{Ric}|^{2}|\mathrm{Rm}|^{p-1-\alpha}\right) f^{a} \phi^{q} \\
= & \int_{M} \Delta\left(|\mathrm{Ric}|^{2}|\mathrm{Rm}|^{p-1-\alpha}\right) f^{a} \phi^{q} \\
& -\int_{M}\left\langle\nabla f, \nabla\left(|\mathrm{Ric}|^{2}|\mathrm{Rm}|^{p-1-\alpha}\right)\right\rangle f^{a} \phi^{q} \\
= & \int_{M}|\operatorname{Ric}|^{2}|\mathrm{Rm}|^{p-1-\alpha} \Delta\left(f^{a} \phi^{q}\right) \\
& +\int_{M}|\operatorname{Ric}|^{2}|\mathrm{Rm}|^{p-1-\alpha}\left(\Delta f+a|\nabla f|^{2} f^{-1}\right) f^{a} \phi^{q} \\
& -\frac{q}{r} \int_{M}|\operatorname{Ric}|^{2}|\operatorname{Rm}|^{p-1-\alpha}|\nabla f|^{2} f^{a} \phi^{q-1} .
\end{aligned}
$$

A direct computation shows that

$$
\begin{aligned}
\Delta\left(f^{a} \phi^{q}\right) & \leq\left(\Delta f^{a}\right) \phi^{q}+f^{a} \Delta \phi^{q} \\
& \leq c p^{2} f^{a-1} \phi^{q-2} .
\end{aligned}
$$

Consequently, we get that

$$
\begin{aligned}
\int_{M}|\mathrm{Ric}|^{2}|\mathrm{Rm}|^{p-1-\alpha} \Delta\left(f^{a} \phi^{q}\right) & \leq c p^{2} \int_{M}|\mathrm{Ric}|^{2}|\mathrm{Rm}|^{p-1-\alpha} f^{a-1} \phi^{q-2} \\
& \leq c p^{2} \int_{M}|\mathrm{Rm}|^{p-1} f^{a-1} \phi^{q-2}+C \\
& \leq \frac{c}{p^{4}} \int_{M}|\operatorname{Rm}|^{p} f^{a} \phi^{q}+c(p) \int_{M} f^{a-p} \phi^{q-2 p}+C \\
& \leq \frac{c}{p^{4}} \int_{M}|\operatorname{Rm}|^{p} f^{a} \phi^{q}+C,
\end{aligned}
$$

where in the last line we have used (2.4) and (2.7) to infer that $\int_{M} f^{a-p} \phi^{q-2 p} \leq C$.

Therefore, it follows from (2.18) that

$$
\begin{aligned}
& \int_{M} \Delta_{f}\left(|\mathrm{Ric}|^{2}|\mathrm{Rm}|^{p-1-\alpha}\right) f^{a} \phi^{q} \\
\leq & c p \int_{M}|\mathrm{Ric}|^{2}|\mathrm{Rm}|^{p-1-\alpha} f^{a} \phi^{q} \\
& +\frac{c}{p^{4}} \int_{M}|\mathrm{Rm}|^{p} f^{a} \phi^{q}+C \\
\leq & \frac{c}{p^{4}} \int_{M}|\mathrm{Rm}|^{p} f^{a} \phi^{q}+c(p) \int_{M}|\operatorname{Ric}|^{p} f^{a} \phi^{q}+C,
\end{aligned}
$$

where we have used Young's inequality to obtain the inequality.

Plugging (2.17) and (2.19) into (2.11), we conclude that 
$(2.20)$

$$
\begin{aligned}
c p^{3} \int_{M}|\nabla \mathrm{Ric}|^{2}|\mathrm{Rm}|^{p-1-\alpha} f^{a} \phi^{q} \leq & \frac{c}{p} \int_{M}|\nabla \mathrm{Rm}|^{2}|\mathrm{Rm}|^{p-3+\alpha} f^{a} \phi^{q} \\
& +\frac{c}{p} \int_{M}|\mathrm{Rm}|^{p} f^{a} \phi^{q} \\
& +c(p) \int_{M}|\mathrm{Ric}|^{p} f^{a} \phi^{q}+C
\end{aligned}
$$

as

$$
c p \int_{M}|\operatorname{Ric}|^{2}|\mathrm{Rm}|^{p-\alpha} f^{a} \phi^{q} \leq \frac{1}{p} \int_{M}|\operatorname{Rm}|^{p} f^{a} \phi^{q}+c(p) \int_{M}|\operatorname{Ric}|^{p} f^{a} \phi^{q}+C .
$$

For the third term on the right hand side of (2.10), we now claim that

$$
\begin{aligned}
& \frac{c p}{r} \int_{M}\left|R_{i j k h} f_{h}\right|^{2}|\mathrm{Rm}|^{p-2} f^{a} \phi^{q-1} \\
\leq & \frac{c}{p} \int_{M}|\nabla \mathrm{Rm}|^{2}|\mathrm{Rm}|^{p-3+\alpha} f^{a} \phi^{q} \\
& +\frac{c}{p} \int_{M}|\mathrm{Rm}|^{p} f^{a} \phi^{q}+C .
\end{aligned}
$$

Indeed, by (2.1),

$$
\begin{aligned}
& \frac{c p}{r} \int_{M}\left|R_{i j k h} f_{h}\right|^{2}|\mathrm{Rm}|^{p-2} f^{a} \phi^{q-1} \\
= & \frac{c p}{r} \int_{M} \nabla_{j} R_{i k}\left(R_{i j k h} f_{h}\right)|\mathrm{Rm}|^{p-2} f^{a} \phi^{q-1} \\
= & -\frac{c p}{r} \int_{M} R_{i k} f_{h}\left(\nabla_{j} R_{i j k h}\right)|\mathrm{Rm}|^{p-2} f^{a} \phi^{q-1} \\
& -\frac{c p}{r} \int_{M} R_{i k} R_{i j k h} f_{h}\left(\nabla_{j}|\mathrm{Rm}|^{p-2}\right) f^{a} \phi^{q-1} \\
& -\frac{c p}{r} \int_{M} R_{i k} f_{h j} R_{i j k h}|\mathrm{Rm}|^{p-2} f^{a} \phi^{q-1} \\
& -\frac{c a p}{r} \int_{M} R_{i k} R_{i j k h} f_{h} f_{j}|\mathrm{Rm}|^{p-2} f^{a-1} \phi^{q-1} \\
& +\frac{c p(q-1)}{r^{2}} \int_{M} R_{i k} R_{i j k h} f_{h} f_{j}|\mathrm{Rm}|^{p-2} f^{a} \phi^{q-2} .
\end{aligned}
$$


The last three terms above can be bounded by

$$
\begin{aligned}
& -\frac{c p}{r} \int_{M} R_{i k} f_{h j} R_{i j k h}|\mathrm{Rm}|^{p-2} f^{a} \phi^{q-1} \\
& -\frac{c a p}{r} \int_{M} R_{i k} R_{i j k h} f_{h} f_{j}|\mathrm{Rm}|^{p-2} f^{a-1} \phi^{q-1} \\
& +\frac{c q p}{r^{2}} \int_{M} R_{i k} R_{i j k h} f_{h} f_{j}|\mathrm{Rm}|^{p-2} f^{a} \phi^{q-2} \\
\leq & \frac{c}{r} \int_{M}|\mathrm{Rm}|^{p-1} f^{a} \phi^{q-2} \\
\leq & \frac{c}{p} \int_{M}|\mathrm{Rm}|^{p} f^{a} \phi^{q}+C .
\end{aligned}
$$

Furthermore, note that

$$
\begin{aligned}
& -\frac{c p}{r} \int_{M} R_{i k} R_{i j k h} f_{h}\left(\nabla_{j}|\mathrm{Rm}|^{p-2}\right) f^{a} \phi^{q-1} \\
& -\frac{c p}{r} \int_{M} R_{i k} f_{h}\left(\nabla_{j} R_{i j k h}\right)|\mathrm{Rm}|^{p-2} f^{a} \phi^{q-1} \\
\leq & \frac{c p^{2}}{\sqrt{r}} \int_{M}|\operatorname{Ric}||\nabla \mathrm{Rm}||\mathrm{Rm}|^{p-2} f^{a} \phi^{q-1} \\
\leq & \frac{1}{p} \int_{M}|\nabla \mathrm{Rm}|^{2}|\mathrm{Rm}|^{p-3+\alpha} f^{a} \phi^{q}+\frac{c(p)}{r} \int_{M}|\mathrm{Ric}|^{2}|\mathrm{Rm}|^{p-1-\alpha} f^{a} \phi^{q-2} .
\end{aligned}
$$

Since

$$
\begin{aligned}
& \frac{c(p)}{r} \int_{M}|\mathrm{Ric}|^{2}|\mathrm{Rm}|^{p-1-\alpha} f^{a} \phi^{q-2} \\
\leq & \frac{1}{p} \int_{M}|\mathrm{Rm}|^{p} f^{a} \phi^{q}+\frac{c(p)}{r^{\frac{p}{1+\alpha}}} \int_{M}|\operatorname{Ric}|^{\frac{2 p}{1+\alpha}} f^{a} \phi^{q-\frac{2 p}{1+\alpha}} \\
\leq & \frac{1}{p} \int_{M}|\operatorname{Rm}|^{p} f^{a} \phi^{q}+C,
\end{aligned}
$$

we see that

$$
\begin{aligned}
& -\frac{c p}{r} \int_{M} R_{i k} R_{i j k h} f_{h}\left(\nabla_{j}|\mathrm{Rm}|^{p-2}\right) f^{a} \phi^{q-1} \\
\leq & \frac{1}{p} \int_{M}|\nabla \mathrm{Rm}|^{2}|\mathrm{Rm}|^{p-3+\alpha} f^{a} \phi^{q}+\frac{1}{p} \int_{M}|\mathrm{Rm}|^{p} f^{a} \phi^{q}+C .
\end{aligned}
$$

These estimates imply that (2.21) is indeed true. Putting (2.20) and (2.21) into (2.10) we conclude that

$$
\begin{aligned}
\int_{M}|\mathrm{Rm}|^{p} f^{a} \phi^{q} \leq & \frac{c}{p} \int_{M}|\nabla \mathrm{Rm}|^{2}|\mathrm{Rm}|^{p-3+\alpha} f^{a} \phi^{q} \\
& +c(p) \int_{M}|\operatorname{Ric}|^{p} f^{a} \phi^{q}+C .
\end{aligned}
$$


We now use that

$$
2|\nabla \mathrm{Rm}|^{2} \leq \Delta|\mathrm{Rm}|^{2}-\left\langle\nabla f, \nabla|\mathrm{Rm}|^{2}\right\rangle+c|\mathrm{Rm}|^{3}
$$

to estimate

$$
\begin{aligned}
2 \int_{M}|\nabla \mathrm{Rm}|^{2}|\mathrm{Rm}|^{p-3+\alpha} f^{a} \phi^{q} \leq & \int_{M}\left(\Delta|\mathrm{Rm}|^{2}\right)|\mathrm{Rm}|^{p-3+\alpha} f^{a} \phi^{q} \\
& -\int_{M}\left\langle\nabla f, \nabla|\mathrm{Rm}|^{2}\right\rangle|\mathrm{Rm}|^{p-3+\alpha} f^{a} \phi^{q} \\
& +c \int_{M}|\mathrm{Rm}|^{p+\alpha} f^{a} \phi^{q} \\
\leq & -a \int_{M}\left\langle\nabla f, \nabla|\mathrm{Rm}|^{2}\right\rangle|\mathrm{Rm}|^{p-3+\alpha} f^{a-1} \phi^{q} \\
& +\frac{q}{r} \int_{M}\left\langle\nabla f, \nabla|\mathrm{Rm}|^{2}\right\rangle|\mathrm{Rm}|^{p-3+\alpha} f^{a} \phi^{q-1} \\
& -\int_{M}\left\langle\nabla f, \nabla|\mathrm{Rm}|^{2}\right\rangle|\mathrm{Rm}|^{p-3+\alpha} f^{a} \phi^{q} \\
& +c \int_{M}|\mathrm{Rm}|^{p+\alpha} f^{a} \phi^{q} .
\end{aligned}
$$

However,

$$
\begin{aligned}
& -\int_{M}\left\langle\nabla f, \nabla|\mathrm{Rm}|^{2}\right\rangle|\mathrm{Rm}|^{p-3+\alpha} f^{a} \phi^{q} \\
= & -\frac{2}{p-1+\alpha} \int_{M}\left\langle\nabla f, \nabla|\mathrm{Rm}|^{p-1+\alpha}\right\rangle f^{a} \phi^{q} \\
= & \frac{2}{p-1+\alpha} \int_{M}\left((\Delta f) f^{a}+a|\nabla f|^{2} f^{a-1}\right)|\mathrm{Rm}|^{p-1+\alpha} \phi^{q} \\
& -\frac{2 q}{p-1+\alpha} \frac{1}{r} \int_{M}|\nabla f|^{2} f^{a}|\mathrm{Rm}|^{p-1+\alpha} \phi^{q-1} \\
\leq & c \int_{M}|\mathrm{Rm}|^{p-1+\alpha} f^{a} \phi^{q} .
\end{aligned}
$$

Similarly,

$$
\begin{aligned}
& -a \int_{M}\left\langle\nabla f, \nabla|\mathrm{Rm}|^{2}\right\rangle|\mathrm{Rm}|^{p-3+\alpha} f^{a-1} \phi^{q} \\
\leq & c p \int_{M}|\mathrm{Rm}|^{p-1+\alpha} f^{a-1} \phi^{q} \\
\leq & c \int_{M}|\mathrm{Rm}|^{p-1+\alpha} f^{a} \phi^{q}+C .
\end{aligned}
$$


Finally, a similar argument implies that

$$
\begin{aligned}
& \frac{q}{r} \int_{M}\left\langle\nabla f, \nabla|\mathrm{Rm}|^{2}\right\rangle|\mathrm{Rm}|^{p-3+\alpha} f^{a} \phi^{q-1} \\
\leq & \frac{c p}{r} \int_{M}|\mathrm{Rm}|^{p-1+\alpha} f^{a} \phi^{q-2}+C \\
\leq & c \int_{M}|\mathrm{Rm}|^{p+\alpha} f^{a} \phi^{q}+\frac{c(p)}{r^{p+\alpha}} \int_{M} f^{a} \phi^{q-2(p+\alpha)}+C \\
\leq & c \int_{M}|\mathrm{Rm}|^{p+\alpha} f^{a} \phi^{q}+C,
\end{aligned}
$$

where we have used (2.7) and (2.4) in the last line. The above estimates show that

$$
\begin{aligned}
\int_{M}|\nabla \mathrm{Rm}|^{2}|\mathrm{Rm}|^{p-2} f^{a} \phi^{q} \leq & c \int_{M}|\mathrm{Rm}|^{p-1+\alpha} f^{a} \phi^{q} \\
& +c \int_{M}|\mathrm{Rm}|^{p+\alpha} f^{a} \phi^{q}+C .
\end{aligned}
$$

Plugging this in (2.22), we arrive at

$$
\begin{aligned}
\int_{M}|\operatorname{Rm}|^{p} f^{a} \phi^{q} \leq & \frac{c}{p} \int_{M}|\operatorname{Rm}|^{p-1+\alpha} f^{a} \phi^{q}+\frac{c}{p} \int_{M}|\operatorname{Rm}|^{p+\alpha} f^{a} \phi^{q} \\
& +c(p) \int_{M}|\operatorname{Ric}|^{p} f^{a} \phi^{q}+C .
\end{aligned}
$$

This proves the lemma.

Using Lemma 2.1 we obtain the following crucial result. From now on, we assume $p \geq 8 n, a \leq \frac{1}{4} p$ and $q \geq 2 p+5$.

Proposition 2.2. Let $(M, g, f)$ be an $n$ dimensional shrinking Ricci soliton with $\lim _{x \rightarrow \infty}|\operatorname{Ric}|(x)=0$. Then

$$
\int_{M}|\operatorname{Rm}|^{p} f^{a} \phi^{q} \leq c(p) \int_{M}|\operatorname{Ric}|^{p} f^{a} \phi^{q}+C .
$$

Proof. Applying Lemma 2.1 for $\alpha=1$ we get

$$
\begin{aligned}
\int_{M}|\mathrm{Rm}|^{p} f^{a} \phi^{q} \leq & \frac{c}{p} \int_{M}|\mathrm{Rm}|^{p+1} f^{a} \phi^{q} \\
& +c(p) \int_{M}|\operatorname{Ric}|^{p} f^{a} \phi^{q}+C .
\end{aligned}
$$

Since $q \geq 2(p+1)+3$, we may apply Lemma 2.1 for $\alpha=0$ and conclude that

$$
\begin{aligned}
\int_{M}|\mathrm{Rm}|^{p+1} f^{a} \phi^{q} \leq & \frac{c}{p} \int_{M}|\operatorname{Rm}|^{p} f^{a} \phi^{q} \\
& +c(p) \int_{M}|\operatorname{Ric}|^{p} f^{a} \phi^{q}+C .
\end{aligned}
$$

Proposition 2.2 follows by combining (2.23) and (2.24).

This proposition enables us to obtain the following bound for Ricci curvature. We continue to assume that $p \geq 8 n, a \leq \frac{1}{4} p$ and $q \geq 2 p+5$. 
Proposition 2.3. Let $(M, g, f)$ be an $n$ dimensional shrinking Ricci soliton with $\lim _{x \rightarrow \infty}|\operatorname{Ric}|(x)=0$. Then

$$
\int_{M}|\operatorname{Ric}|^{p} f^{a}<\infty
$$

Proof. Recall that

$$
\Delta R_{i j}-\left\langle\nabla R_{i j}, \nabla f\right\rangle=R_{i j}-2 R_{i k j l} R_{i j} R_{k l} .
$$

This implies that

$$
\left.\mid \text { Ric }\left.\right|^{2} \leq \frac{1}{2} \Delta \mid \text { Ric }\left.\right|^{2}-\frac{1}{2}\langle\nabla f, \nabla| \text { Ric }\left.\right|^{2}\right\rangle+2 \mid \text { Rm }|| \text { Ric }\left.\right|^{2}-\mid \nabla \text { Ric }\left.\right|^{2} .
$$

Therefore,

$$
\begin{aligned}
\int_{M}|\operatorname{Ric}|^{p} f^{a} \phi^{q}= & \frac{1}{2} \int_{M} \Delta|\operatorname{Ric}|^{2}|\operatorname{Ric}|^{p-2} f^{a} \phi^{q} \\
& -\frac{1}{2} \int_{M}\left\langle\nabla f, \nabla|\operatorname{Ric}|^{2}\right\rangle|\operatorname{Ric}|^{p-2} f^{a} \phi^{q} \\
& +2 \int_{M}|\operatorname{Rm}||\operatorname{Ric}|^{p} f^{a} \phi^{q} \\
& -\int_{M}|\nabla \operatorname{Ric}|^{2}|\operatorname{Ric}|^{p-2} f^{a} \phi^{q} .
\end{aligned}
$$

Integrating by parts, we get

$$
\begin{aligned}
\frac{1}{2} \int_{M} \Delta|\mathrm{Ric}|^{2}|\mathrm{Ric}|^{p-2} f^{a} \phi^{q} \leq & -\frac{a}{2} \int_{M}\left\langle\nabla f, \nabla|\mathrm{Ric}|^{2}\right\rangle|\mathrm{Ric}|^{p-2} f^{a-1} \phi^{q} \\
& +\frac{q}{2 r} \int_{M}\left\langle\nabla f, \nabla|\mathrm{Ric}|^{2}\right\rangle|\mathrm{Ric}|^{p-2} f^{a} \phi^{q-1} \\
\leq & c p \int_{M}|\nabla \mathrm{Ric}||\mathrm{Ric}|^{p-1} f^{a-\frac{1}{2}} \phi^{q-1} \\
\leq & \int_{M}|\nabla \mathrm{Ric}|^{2}|\operatorname{Ric}|^{p-2} f^{a} \phi^{q}+c p^{2} \int_{M}|\operatorname{Ric}|^{p} f^{a-1} \phi^{q-2} .
\end{aligned}
$$

However, 2.5D and Young's inequality imply

$$
\begin{aligned}
c p^{2} \int_{M}|\operatorname{Ric}|^{p} f^{a-1} \phi^{q-2} & \leq \frac{c}{p} \int_{M}|\operatorname{Ric}|^{p-1} f^{a-1} \phi^{q-2}+C \\
& \leq \frac{1}{p} \int_{M}|\operatorname{Ric}|^{p} f^{a} \phi^{q}+C .
\end{aligned}
$$

This proves that

$$
\begin{aligned}
\frac{1}{2} \int_{M} \Delta|\mathrm{Ric}|^{2}|\mathrm{Ric}|^{p-2} f^{a} \phi^{q} \leq & \int_{M}|\nabla \mathrm{Ric}|^{2}|\mathrm{Ric}|^{p-2} f^{a} \phi^{q} \\
& +\frac{1}{p} \int_{M}|\mathrm{Ric}|^{p} f^{a} \phi^{q}+C .
\end{aligned}
$$

According to Proposition 2.2, there exists a constant $c_{0}(p)>0$ so that

$$
\int_{M}|\operatorname{Rm}|^{p} f^{a} \phi^{q} \leq c_{0}(p) \int_{M}|\operatorname{Ric}|^{p} f^{a} \phi^{q}+C .
$$


For this constant $c_{0}(p)$, we use Young's inequality to conclude

$$
\begin{aligned}
\int_{M}|\operatorname{Rm}||\operatorname{Ric}|^{p} f^{a} \phi^{q} \leq & \frac{1}{p} \frac{1}{c_{0}(p)} \int_{M}|\operatorname{Rm}|^{p} f^{a} \phi^{q} \\
& +c_{1}(p) \int_{M}|\operatorname{Ric}|^{\frac{p^{2}}{p-1}} f^{a} \phi^{q} \\
\leq & \frac{1}{p} \int_{M}|\operatorname{Ric}|^{p} f^{a} \phi^{q} \\
& +c_{1}(p) \int_{M}|\operatorname{Ric}|^{p+\frac{p}{p-1}} f^{a} \phi^{q}+C .
\end{aligned}
$$

Since $\lim _{x \rightarrow \infty}|\operatorname{Ric}|=0$, there exists $r_{1}>0$ so that on $M \backslash D\left(r_{1}\right)$

$$
\mid \text { Ric }\left.\right|^{\frac{p}{p-1}} \leq \frac{1}{p c_{1}(p)}
$$

Hence, (2.27) yields that

$$
\int_{M}|\operatorname{Rm}||\operatorname{Ric}|^{p} f^{a} \phi^{q} \leq \frac{2}{p} \int_{M}|\operatorname{Ric}|^{p} f^{a} \phi^{q}+C,
$$

where $C$ depends on the geometry of $D\left(r_{1}\right)$.

Using (2.26) and (2.28) in (2.25) implies that

$$
\left.\int_{M}|\operatorname{Ric}|^{p} f^{a} \phi^{q} \leq-\int_{M}\langle\nabla f, \nabla| \text { Ric }\left.\right|^{2}\right\rangle|\operatorname{Ric}|^{p-2} f^{a} \phi^{q}+C .
$$

However, as $a \leq \frac{p}{4}$ and $p \geq 8 n$, we get

$$
\begin{aligned}
-\int_{M}\left\langle\nabla f, \nabla|\operatorname{Ric}|^{2}\right\rangle|\operatorname{Ric}|^{p-2} f^{a} \phi^{q} & =-\frac{2}{p} \int_{M}\left\langle\nabla f, \nabla|\operatorname{Ric}|^{p}\right\rangle f^{a} \phi^{q} \\
& \leq \frac{2}{p} \int_{M}|\operatorname{Ric}|^{p}\left((\Delta f) f^{a}+a|\nabla f|^{2} f^{a-1}\right) \phi^{q} \\
& \leq \frac{2}{3} \int_{M}|\operatorname{Ric}|^{p} f^{a} \phi^{q} .
\end{aligned}
$$

Together with (2.29), we obtain

$$
\int_{M}|\operatorname{Ric}|^{p} f^{a} \phi^{q} \leq C
$$

Combining Proposition 2.2 with Proposition 2.3 one concludes that

$$
\int_{M}|\mathrm{Rm}|^{p} f^{a} \leq C
$$

We are now ready to prove the main theorem of the paper.

Theorem 2.4. Let $(M, g, f)$ be a gradient shrinking Ricci soliton of dimension $n$ with Ricci curvature convergent to zero at infinity. Then $(M, g, f)$ is $C^{k}$ asymptotic to a cone for all $k$.

Proof. Applying (2.30) for $a=\frac{p}{4}$ implies that

$$
\int_{B_{x}(1)}|\mathrm{Rm}|^{p} \leq C\left(d\left(x_{0}, x\right)+1\right)^{-\frac{p}{2}}
$$


Note that

$$
\Delta|\mathrm{Rm}|^{2} \geq-u|\mathrm{Rm}|^{2},
$$

where $u:=c(|\mathrm{Rm}|+f)$. Furthermore, the Sobolev constant of $B_{x}(1)$ depends only on dimension, the Ricci curvature bound and Perelman's invariant 21. So the Moser iteration [18] implies that

$$
\begin{aligned}
|\operatorname{Rm}|(x) & \leq C\left(\int_{B_{x}(1)} u^{n}+1\right)^{\frac{1}{p}}\left(\int_{B_{x}(1)}|\mathrm{Rm}|^{p}\right)^{\frac{1}{p}} \\
& \leq C\left(d\left(x_{0}, x\right)+1\right)^{-\frac{1}{4}} .
\end{aligned}
$$

In particular, this shows that $\lim _{x \rightarrow \infty}|\mathrm{Rm}|=0$. Now from

$$
\Delta_{f}|\mathrm{Rm}| \geq|\mathrm{Rm}|-c|\mathrm{Rm}|^{2}
$$

and the information that $|\mathrm{Rm}| \rightarrow 0$ at infinity, it follows that $|\mathrm{Rm}|$ decays quadratically, that is,

$$
|\mathrm{Rm}|(x) \leq c\left(d\left(x_{0}, x\right)+1\right)^{-2} .
$$

Indeed, it was shown in [20] that if a nonnegative function $w$ satisfies

$$
\Delta_{f} w \geq w-c w^{2}
$$

on a shrinking Ricci soliton and $w \rightarrow 0$ at infinity, then there exists a constant $c>0$ so that $w \leq \frac{c}{f}$. The proof given in [20] is in dimension $n=4$, but it is easy to see that the same argument works in any dimension.

Now (2.31) and Shi's derivative estimates imply the derivatives of the Riemann curvature tensor satisfy

$$
\left|\nabla^{k} \mathrm{Rm}\right|(x) \leq c\left(d\left(x_{0}, x\right)+1\right)^{-k-2}
$$

for all $k \geq 1$. From this, it follows that $(M, g)$ is $C^{k}$ asymptotic to a cone for all $k$. We refer to [17 for more details. The theorem is proved.

\section{REFERENCES}

[1] C. Böhm and B. Wilking, Manifolds with positive curvature operators are space forms, Ann. Math. (2) 167 (2008), no. 3, 1079-1097.

[2] H. D. Cao, Existence of gradient Kähler-Ricci solitons, Elliptic and parabolic methods in geometry (B. Chow, R. Gulliver, S. Levy, J. Sullivan, editors), AK Peters, 1996, 1-16.

[3] H.D. Cao, Recent progress on Ricci solitons, Adv. Lect. Math. 11, no. 2, 1-38, Int. Press, Somerville, MA, 2010.

[4] H.D. Cao, Geometry of complete gradient shrinking Ricci solitons, Adv. Lect. Math., 17, no. 1, 227-246, Int. Press, Somerville, MA, 2011.

[5] H. D. Cao, B. L. Chen, X. P. Zhu, Recent developments on Hamilton's Ricci flow. Surveys in differential geometry. Vol. XII. Geometric flows, 47-112, Surv. Differ. Geom., 12, Int. Press, Somerville, MA, 2008.

[6] H. D. Cao and Q. Chen, On Bach-flat gradient shrinking Ricci solitons, Duke Math. J. 162 (2013), no.6, 1149-1169.

[7] H.D. Cao and D. Zhou, On complete gradient shrinking Ricci solitons, J. Differential Geom. 85 (2010), no. 2, 175-186.

[8] X. Cao, B. Wang and Z. Zhang, On locally conformally flat gradient shrinking Ricci solitons, Commun. Contemp. Math. 13 (2011), no. 2, 269-282.

[9] B.L. Chen, Strong uniqueness of the Ricci flow, J. Differential Geom. 82 (2009), no. 2, 362-382.

[10] B. Chow, P. Lu and L. Ni, Hamilton's Ricci flow. Graduate Studies in Mathematics, 77. American Mathematical Society, Providence, RI; Science Press, New York, 2006. 
[11] A. Dancer and M. Wang, On Ricci solitons of cohomogeneity one, Ann. Global Anal. Geom. 39 (2011), no. 3, 259-292.

[12] M. Eminenti, G. La Nave and C. Mantegazza, Ricci solitons: the equation point of view, Manuscripta Math. 127 (2008), 345-367.

[13] J. Enders, R. Müller, P. Topping, On Type-I singularities in Ricci flow, Comm. Anal. Geom, 19 (2011), no. 5, 905-922.

[14] M. Feldman, T. Ilmanen and D. Knopf, Rotationally symmetric shrinking and expanding gradient Kähler-Ricci solitons, J. Differential Geom. 65 (2003), no. 2, 169-209.

[15] R. Hamilton, The formation of singularities in the Ricci flow, Surveys in Differential Geom. 2 (1995), 7-136, International Press.

[16] N. Koiso, On rotationally symmetric Hamilton's equation for Kähler-Einstein metrics, Advanced Studies in Pure Math., 18 (1), 327-337, Kinokuniya (Tokyo) and Academic Press (Boston), 1990.

[17] B. Kotschwar and L. Wang, Rigidity of Asymptotically Conical Shrinking Gradient Ricci Solitons, J. Differential Geom. (to appear).

[18] P. Li, Geometric Analysis, Cambridge Studies in Advanced Mathematics, 2012.

[19] O. Munteanu and N. Sesum, On gradient Ricci solitons, J. Geom. Anal. 23 (2013), no. 2, 539-561.

[20] O. Munteanu and J. Wang, Geometry of shrinking Ricci solitons, arXiv:1410.3813

[21] O. Munteanu and M.T. Wang, The curvature of gradient Ricci solitons, Math. Res. Lett. 18 (2011), no. 6, 1051-1069.

[22] A. Naber, Noncompact Shrinking 4-Solitons with Nonnegative Curvature, J. Reine Angew. Math., 645 (2010), 125-153.

[23] L. Ni and N. Wallach, On a classification of gradient shrinking solitons, Math. Res. Lett. 15 (2008), no. 5, 941-955.

[24] G. Perelman, The entropy formula for the Ricci flow and its geometric applications, arXiv:math. DG/0211159.

[25] P. Petersen and P. Wylie, On the classification of gradient Ricci solitons, Geom. Topol. 14 (2010), 2277-2300.

[26] W. X. Shi, Deforming the metric on complete Riemannian manifolds, J. Differential Geom. 30 (1989), no. 1, 223-301.

[27] X.J. Wang and X. Zhu, Kähler-Ricci solitons on toric manifolds with positive first Chern class, Adv. Math. 188 (2004), 87-103.

[28] Z. H. Zhang, Gradient shrinking solitons with vanishing Weyl tensor, Pacific J. Math. 242 (2009), 189-200.

E-mail address: ovidiu.munteanu@uconn.edu

Department of Mathematics, University of Connecticut, Storrs, CT 06268, USA

E-mail address: jiaping@math.umn.edu

School of Mathematics, University of Minnesota, Minneapolis, MN 55455, USA 